\title{
Arthritis in mice induced by a single immunisation with collagen
}

\author{
Fuminori Kato, Masanao Nomura, Kyoko Nakamura
}

\begin{abstract}
Objective-To determine whether collagen induced arthritis (CIA) in mice can be satisfactorily induced by a single immunisation and whether this model has some advantages compared with conventional CIA, which is induced by two immunisations.

Methods-The incidence of arthritis was observed under different immunisation conditions (variation of species of Mycobacterium included in complete Freund's adjuvant and the method of emulsification) and immunological, histopathological, and pharmacological features were examined.

Results-Under optimum immunisation conditions, joint inflammation developed two to three weeks after the primary immunisation with an incidence of $100 \%$ at four to five weeks. The progression of the arthritis was mild and was associated with moderate increases in concentrations of serum IgG against type II collagen. This CIA model was similar to the conventional model in histopathological and pharmacological features.

Conclusions-Murine CIA could be successfully induced by a single immunisation. An important feature of this model was a mild progression of joint inflammation. This feature seems to be of benefit for monitoring the development of arthritis from an early stage in the disease and for the development of novel antirheumatic drugs for such early stage patients.
\end{abstract}

(Ann Rheum Dis 1996; 55: 535-539)

Rheumatoid arthritis remains a serious autoimmune disease, despite progress in its management. Therefore, new drugs for treatment of rheumatoid arthritis have been developed worldwide. The rat model of adjuvant induced arthritis (AIA) made an important contribution to the evaluation of antirheumatic drugs, ${ }^{1}$ leading to the development of many non-steroidal anti-inflammatory drugs (NSAIDs) and disease modifying antirheumatic drugs (DMARDs). However, a cure for rheumatism was still elusive and the conventional animal model had probably already reached a limit of usefulness in the development of new drugs; the development of new experimental animal models was necessary.
In 1977 and 1980, experimental models of chronic inflammatory arthritis were developed in rats $^{2}$ and mice. ${ }^{3}$ These collagen induced arthritis (CIA) models resembled human rheumatoid arthritis. ${ }^{2-4}$ Arthritis in rats has been induced by a single injection of type II collagen emulsified in Freund's incomplete adjuvant. The joint inflammation develops two to three weeks after the primary sensitisation. On the other hand, arthritis in mice has usually been induced by two injections of type II collagen emulsified in Freund's complete or incomplete adjuvant. The joint inflammation develops four to five weeks after the primary sensitisation. The susceptibility of mice to arthritis and immune responses to collagen are under the control of the major histocompatibility complex. ${ }^{5}$ As traditional NSAIDs and DMARDs had no effect on joint inflammation in this mouse model, it could be applied to the discovery of novel immunosuppressive agents. ${ }^{6}$

Although there are many studies on the mouse model, no attempt has so far been made to explain why the secondary immunisation is generally required to induce arthritis in mice. Therefore, we examined whether the incidence of arthritis could be improved by modifications of the immunisation conditions, based on the idea that an autoimmune disease should be induced by a single inoculation if it is satisfactorily performed. The autoimmune disease should develop more naturally than when artificial stimulations are repeated. Here we used immunisation conditions in which mouse arthritis could be induced with one inoculation, and report the usefulness of this model for the development of new drugs.

\section{Materials and methods}

ANIMALS

DBA/1JN Crj mice (4 weeks old) were supplied by Charles River Japan Inc, Kanagawa, Japan. They were used for the experimental protocols after a resting period of two to three weeks.

\section{CHEMICAL REAGENTS}

Dexamethasone (Wako Pure Chemical Industries, Osaka, Japan; No 041-18861) was dissolved in dimethylsulphoxide (DMSO) and diluted with physiological saline. Cyclosporin A (Sandimmun: Sandoz, Tokyo, Japan) was diluted with physiological saline. Aspirin 
(synthesised in our laboratory), lobenzarit disodium (Chugai Pharmaceutical Co, Tokyo, Japan), and auranofin (Smith Kline-Fujisawa Co, Tokyo, Japan) were suspended in physiological saline containing $1 \%$ DMSO and $1 \%$ Tween 80 .

\section{INDUCTION OF ARTHRITIS}

Male DBA/1J mice were given an emulsion of bovine collagen type II (C II; $150 \mu \mathrm{g} / 0.1 \mathrm{ml} /$ mouse, Collagen Gijutsu-kenshukai, Japan; No K41) in an equal volume of Freund's complete adjuvant at the base of the tail. Freund's adjuvant containing $0.5 \mathrm{mg} / \mathrm{ml}$ Mycobacterium tuberculosis H37Ra, (ICN Immunobiologicals, Costa Mesa, CA, USA; No $64-285-1$ ) or $0.5 \mathrm{mg} / \mathrm{ml}$ Mycobacterium butyricum (Difco Laboratories, Detroit, Michigan, USA; No 638-60-7) was used. The emulsification was done with an ultrasonicator (Sonicator W-225, Heat System-Ultrasonics, NY, USA) or with connected syringes. The ultrasonication was performed on ice, three times for 20 seconds each time, at intervals of one minute.

\section{EVALUATION OF ARTHRITIS}

The mice were examined weekly throughout the test. The degree of arthritis was represented by scores from 0 to 3 points per foot, maximum of 12 points as the total score of four feet: 0 points: no change; 1 point: detectable swelling in one or more digital joints; 2 points: mild swelling in one or more joints; 3 points: severe swelling of the entire paw or ankylosis.

\section{MEASUREMENT OF ANTIBODY}

CONCENTRATIONS

Serum antibody titres were measured by modification of an enzyme linked immunosorbent assay (ELISA) described previously. ${ }^{7}$ Firstly, the 96 well microplate was coated with $50 \mu \mathrm{l} /$ well of a $10 \mu \mathrm{g} / \mathrm{ml}$ solution of C II at room temperature for 60 minutes, and then blocked with PBS containing $0 \cdot 1 \%$ BSA at room temperature for 60 minutes. Then the diluted serum $(1: 2500)$ was added at $50 \mu \mathrm{l} /$ well and allowed to react at room temperature for 60 minutes. An alkaline phosphatase labelled rabbit antimouse IgG antibody (Zymed Laboratories Inc, San Francisco, CA, USA; No 61-6622) diluted 1000 times was added at $50 \mu \mathrm{l} /$ well, and allowed to react at room temperature for 60 minutes. The wells were washed with PBS three times, then a $10 \%$ diethanolamine buffer solution ( $\mathrm{pH} 9.8$ ) containing $1 \mathrm{mg} / \mathrm{ml}$ of p-nitrophenyl phosphate (Sigma Chemical Company, St Louis, MO, USA; No 104-105) was added at $100 \mu 1 /$ well, and allowed to react at room temperature. The absorbance was measured at $405 \mathrm{~nm}$ with a microplate reader (Bio-Rad, Richmond, CA, USA; No 2550). The amount of each antibody was calculated from the calibration curve of the standard antibody.
HISTOLOGICAL STUDY

The mice were killed with an overdose of pentobarbitone. Their legs were removed and fixed in $10 \%$ neutral buffered formalin. The tissues were decalcified in 5\% EDTA- $2 \mathrm{Na}$ solution. The joints were then imbedded in paraffin. The specimens were cut into $6 \mu \mathrm{m}$ sections and stained with haematoxylin and eosin.

\section{STATISTICS}

Differences were evaluated for significance with Dunnett's multiple comparison test or the Aspin-Welch test after Bartlett's test for homogeneity of variance.

\section{Results and discussion}

Collagen induced arthritis can be generated by a single immunisation with C II. On the other hand, CIA in mice is generally believed to require a second immunisation. Why reimmunisation is required to induce arthritis in mice has not been reported. Our study started from our idea that autoimmune diseases could be induced by a single immunisation if it was satisfactorily performed. Pathogenetically, this represents a more natural model of autoimmune disease than when induction by reimmunisation is required.

Firstly, we examined under which conditions induction of arthritis was more efficient, focusing on two points to which little attention has been paid previously - the species of Mycobacterium included in Freund's complete adjuvant, and the method of emulsification of antigen with the adjuvant. We investigated whether there were any differences in the incidence of arthritis between mice inoculated with Freund's complete adjuvant containing $M$ tuberculosis and those injected with the same adjuvant containing $M$ butyricum. We also determined which method of emulsification - ultrasonication or connected syringes - was more efficient for induction of arthritis. Table 1 shows the time course of induction of arthritis by these modified immunisation procedures. The most efficient condition was immunisation with an ultrasonic emulsion of C II in Freund's complete adjuvant containing $M$ tuberculosis. Under these conditions, the time of onset was two to three weeks and the incidence was $100 \%$ at four to five weeks. Under all other conditions examined, the incidence was less and the time of onset was prolonged. This selection of species of Mycobacterium as well as the method of emulsification of antigen with Freund's complete adjuvant seem important for successful induction of arthritis with a single immunisation.

The importance of IgG antibodies against C II in initiating arthritis has been shown. ${ }^{8}$ Therefore, the concentrations of serum antiC II IgG were measured two weeks after the first immunisation, in the early stage of arthritis. Figure 1 shows that the most efficient conditions (ultrasonic emulsification and $M$ 
Table 1 Time course of incidence of arthritis in $D B A / 1 \mathcal{F}$ mice induced by modified immunisations

\begin{tabular}{|c|c|c|c|c|c|c|}
\hline \multirow{2}{*}{$\begin{array}{l}\text { Species of } \\
\text { Mycobacterium }\end{array}$} & \multirow{2}{*}{$\begin{array}{l}\text { Method of } \\
\text { emulsification }\end{array}$} & \multicolumn{5}{|c|}{ Weeks after immunisation } \\
\hline & & 1 & 2 & 3 & 4 & 5 \\
\hline $\begin{array}{l}\text { Experiment } 1 \text { : } \\
M \text { tuberculosis } \\
M \text { tuberculosis } \\
M \text { butyricum }\end{array}$ & $\begin{array}{l}\text { Ultrasonication } \\
\text { Syringe } \\
\text { Ultrasonication }\end{array}$ & $\begin{array}{l}0 / 5 \\
0 / 5 \\
0 / 5\end{array}$ & $\begin{array}{l}0 / 5 \\
0 / 5 \\
0 / 5\end{array}$ & $\begin{array}{l}3 / 5 \\
0 / 5 \\
0 / 5\end{array}$ & $\begin{array}{l}5 / 5 \\
2 / 5 \\
0 / 5\end{array}$ & $\begin{array}{l}5 / 5 \\
3 / 5 \\
2 / 5\end{array}$ \\
\hline $\begin{array}{l}\text { Experiment 2: } \\
M \text { tuberculosis } \\
M \text { tuberculosis } \\
M \text { butyricum } \\
M \text { butyricum }\end{array}$ & $\begin{array}{l}\text { Ultrasonication } \\
\text { Syringe } \\
\text { Ultrasonication } \\
\text { Syringe }\end{array}$ & $\begin{array}{l}0 / 10 \\
0 / 10 \\
0 / 10 \\
0 / 10\end{array}$ & $\begin{array}{l}2 / 10 \\
0 / 10 \\
0 / 10 \\
0 / 10\end{array}$ & $\begin{array}{l}6 / 10 \\
1 / 10 \\
0 / 10 \\
0 / 10\end{array}$ & $\begin{array}{r}10 / 10 \\
5 / 10 \\
3 / 10 \\
0 / 10\end{array}$ & $\begin{array}{r}10 / 10 \\
8 / 10 \\
7 / 10 \\
7 / 10\end{array}$ \\
\hline
\end{tabular}

Ultrasonication was performed on ice three times for $20 \mathrm{~s}$ each time at intervals of one minute to make a fine and uniform emulsion. tuberculosis) to induce IgG antibody coincided with those for the most efficient induction of arthritis (table 1) supporting the importance of anti-C II IgG in the CIA model. Ultrasonication may be useful for rapid production of a fine and uniform emulsion $(\sim 1$ $\mu \mathrm{m})$, which may be essential for reproducible induction of a large IgG antibody. It is difficult to make an emulsion of high quality with connected syringes and the procedure sometimes takes a long time. Thus ultrasonication was superior to the connected syringes for effective activation of humoral immunity as well as induction of arthritis.

The selection of species of Mycobacterium in CFA was important for efficient induction of IgG antibody against C II. At present we have no direct evidence to explain why $M$ tuberculosis is more useful for the induction of $\operatorname{IgG}$ than $M$ butyricum. Mycobacteria in adjuvant seem to contribute to the immunopathological events of CIA in mice, because the mice responded less well to Freund's incomplete adjuvant. Ellis

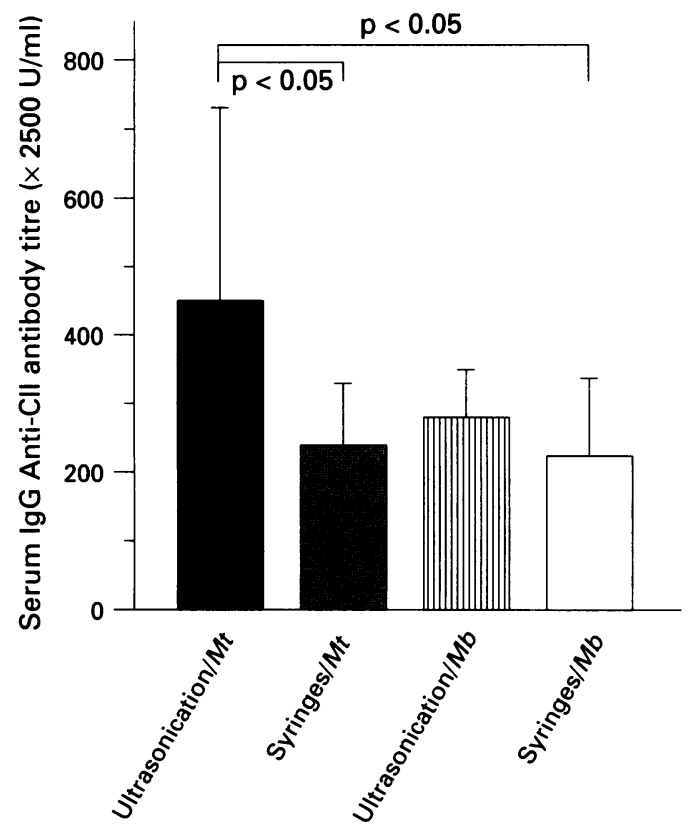

Figure 1 Comparison of humoral responses of mice induced by modified immunisation conditions. Serum samples obtained two weeks after primary immunisation were assayed by ELISA. The titre of antibody to C II was calculated from the calibration curve of standard antibody. calculated from the calibration curve of standard $M t=$ Freund's complete adjuvant with $M$ tuberculosis; $M b=$ Freund's complete adjuvant with $M$ butyricum. et al showed the importance of adjuvant composition in the induction of CIA in mice. ${ }^{10}$ Although $M$ tuberculosis induced a potent $\mathrm{T}$ cell response against $\mathrm{C} \mathrm{II}$, by contrast $M$ butyricum did not. This finding also supports the previous observation that the development of the full pattern of CIA requires synergistic activation of both humoral and cell mediated responses. ${ }^{11}$ Recently, Germann et al reported that interleukin-12 (IL-12) could replace $\mathrm{Myco-}$ bacteria and cause severe arthritis in mice when given in combination with C II. ${ }^{12}$ This cytokine stimulates interferon- $\gamma$ (IFN- $\gamma$ ) synthesis and strongly enhances Th1 cell development. ${ }^{13} 14$ Further studies on cytokines such as IL-12 and IFN- $\gamma$ relating to inflammation and immune responses may be necessary to elucidate the relation between incidence of CIA and the species of mycobacteria. Small mycobacterial fragments are known to be efficient at inducing adjuvant arthritis. ${ }^{15}$ If the resistance of the cell wall to ultrasonication differs between $M$ tuberculosis and $M$ butyricum, it is possible that the degree of arthritis may be dependent on the mycobacterial particle size after ultrasonication. At present, we have no data on particle size. How differences in species or particle size of Mycobacteria in the adjuvant influence immunising capability is a topic for future study.

Ultrasonication can induce heating of samples. However, the temperature of the antigen should not be allowed to become excessively high. Immunisation of mice with heat denatured collagen results in the failure of induction of inflammatory arthritis, ${ }^{11} 16$ and this was associated with the lack of antibodies against conformational determinants present selectively on native antigen. ${ }^{16}$ Therefore, in this study, the ultrasonication was performed on ice. It is important to make a high quality emulsion without a temperature increase. These findings showed that the incidence of mouse arthritis could be altered by slight modifications of the immunisation conditions, and that arthritis could be reproducibly and sufficiently induced by a single immunisation under the conditions described above. The immunisation conditions used here seem to be widely applicable to other models of autoimmune diseases as well as to efficient collection of IgG antibody from the inoculated animal.

Histopathological and pharmacological features of this CIA model were examined. Histopathologically, the arthritic lesions showed synovial proliferation, infiltration with mononuclear cells and granulocytes, fibrin deposits, pannus formation with damage to the cartilage, and bone erosion (fig 2). These features were similar to those of human rheumatoid arthritis and the conventional murine CIA. ${ }^{2-4}$ On the other hand, as conventional NSAIDs and DMARDs have little effect on joint inflammation in the conventional CIA model, the conventional model may be used to characterise novel immunosuppressive agents. ${ }^{6}$ Accordingly, the effects of conventional antirheumatic drugs on this CIA model were examined. As shown in 

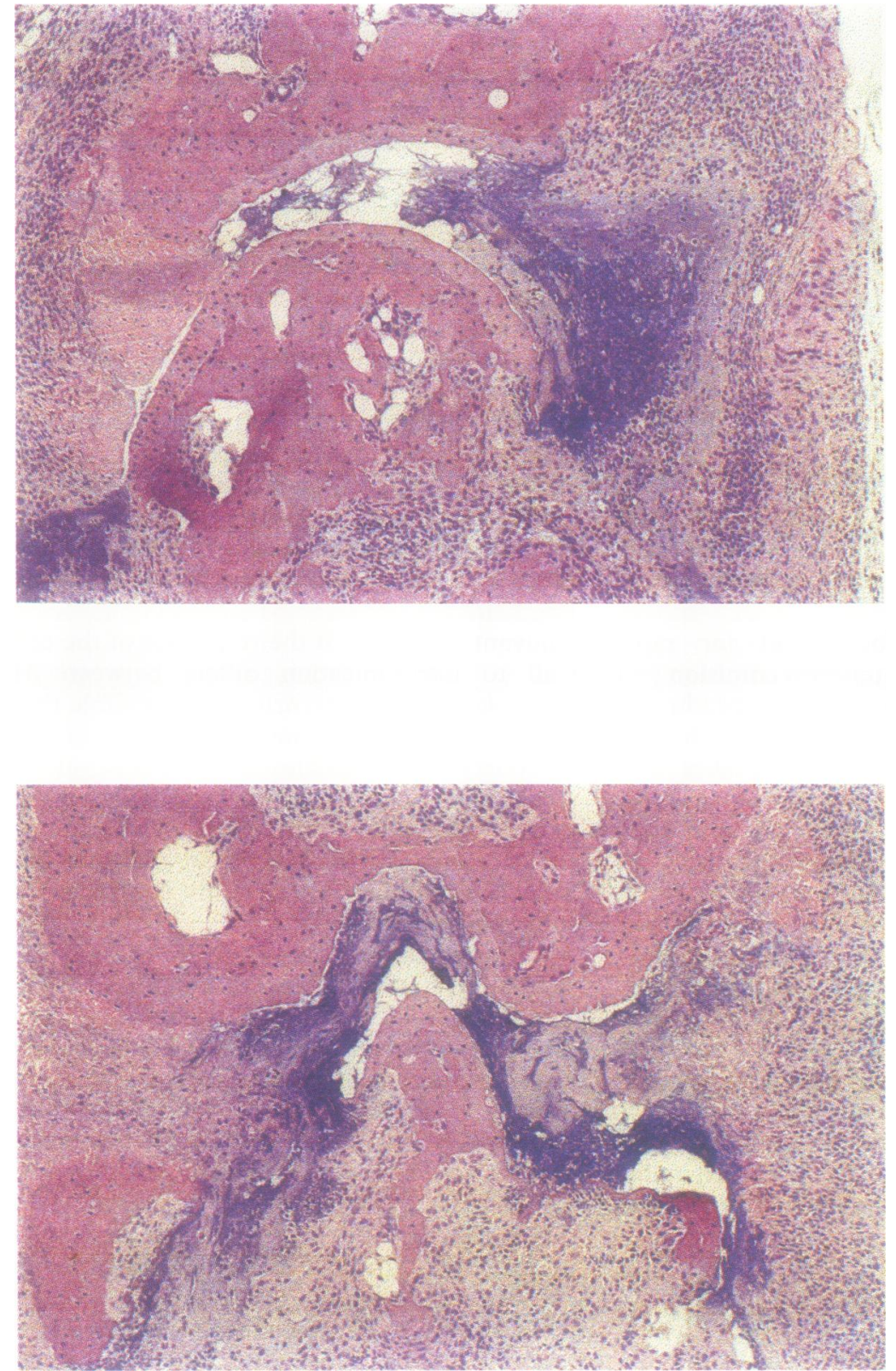

Figure 2 Histopathological findings from the forefeet (top) and hindfeet (bottom) of mice with arthritis. The lesions show synovial proliferation, infiltration with mononuclear cells and granulocytes, fibrin deposits, pannus formation with damage to the cartilage, and bone erosions. Haematoxylin and eosin stain (originally $\times 25$ ).

table 2 , the effects of various agents on this model were similar to those in the conventional CIA model; DMARDs such as lobenzarit and auranofin, and the NSAID aspirin had little suppressive effect on joint inflammation, but the steroidal drug dexamethasone and an immunosuppressive drug cyclosporin A had pronounced suppressive effects. Therefore, this model, in addition to the conventional model, may be used to discover and develop novel antirheumatic agents.

As mentioned, the features of this model resembled those of the conventional CIA model. The distinctive feature of this model was, as shown in fig 3, that the progression of the joint inflammation was mild after small increases in concentration of IgG antibody against C II in serum. On the other hand, when conventionally reimmunised, radical progression of arthritis was found, with a rapid increase in concentration of serum IgG against
Table 2 Effects of antiarthritic drugs on CIA in mice

\begin{tabular}{lcll}
\hline Antiarthritic drug & $\begin{array}{l}\text { Dose } \\
\text { (mg/kg/day) }\end{array}$ & $\begin{array}{l}\text { Incidence of } \\
\text { arthritis in } \\
\text { mice } \\
\text { (n/total) }\end{array}$ & $\begin{array}{l}\text { Arthritis score } \\
\text { (mean (SD) }\end{array}$ \\
\hline Experiment 1: & & & \\
$\quad$ Positive control & - & $4 / 4$ & $4 \cdot 3(2 \cdot 9)$ \\
Lobenzarit & 50 & $4 / 4$ & $5 \cdot 8(2 \cdot 1)$ \\
$\quad$ Auranofin & 10 & $4 / 4$ & $5 \cdot 0(2 \cdot 7)$ \\
Experiment 2: & & & \\
$\quad$ Positive control & - & $5 / 5$ & $6 \cdot 0(1 \cdot 4)$ \\
Aspirin & 200 & $3 / 4$ & $4 \cdot 5(3 \cdot 0)$ \\
Cyclosporin A & 50 & $2 / 4$ & $0 \cdot 8(1 \cdot 0)^{\star}$ \\
Dexamethasone & $0 \cdot 2$ & $1 / 4$ & $0 \cdot 3(0 \cdot 5)^{\star}$ \\
\hline
\end{tabular}

Mice were dosed with the drugs daily for four weeks, starting two weeks after immunisation. Physiological saline was given to the controls in the same manner. All four limbs of each mouse were scored for arthritis on the day after the drug was last given Drugs were given by mouth except for dexamethasone, which was given by intraperitoneal route. ${ }^{\star} \mathrm{p}<0.001 v$ positive control.

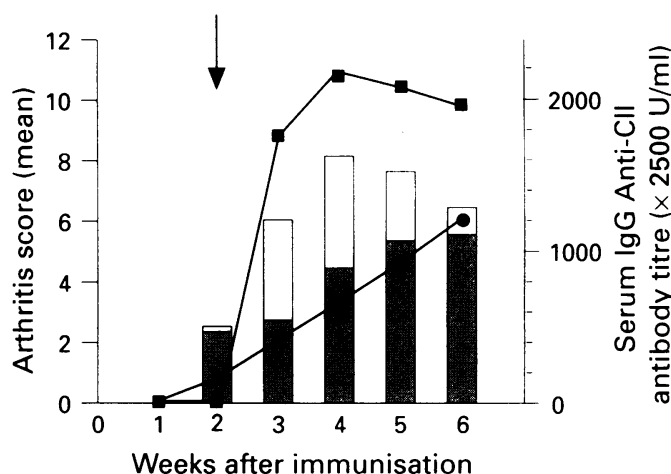

Figure 3 Time course of the arthritis score and humoral response induced by one or two immunisations with C II. Closed circle and striped bar: mean of the arthritis score and the mean of the titre of antibody to CII of 10 mice induced by one immunisation respectively; closed square and open bar: mean of the arthritis score and mean of the titre of antibody to $C$ II of five mice induced by second immunisation at two weeks (arrow).

C II. This phenomenon is a matter of course in secondary immune responses against a foreign antigen, but seems not to be pathogenetically natural as a model of autoimmune disease. Autoantigens which are always present in the body should be able to provide continuous stimuli to immune systems. Accordingly, the second immunisation should be theoretically unnecessary for induction of autoimmunity if the first inoculation with antigen is performed satisfactorily. The successful induction of arthritis such as in this CIA model supports this idea.

This model has some advantages over the conventional CIA model. Firstly, as arthritis can be induced by only one immunisation, it will be more economical, saving time, manpower, and cost. Secondly, joint inflammation develops two to three weeks after the primary immunisation (similarly to the rat CIA model) and the incidence is $100 \%$ at four to five weeks. This time is convenient for evaluation of drugs using a model of chronic autoimmune disease. Thirdly, the progression of arthritis is mild. This is a beneficial feature for a model of chronic disease, and may be useful for the discovery of more extensive antirheumatic agents. Also, the importance of immunotherapy in the early stage of rheumatoid arthritis has been stressed 
recently. ${ }^{17}$ Thus this model will be more helpful in the discovery and development of drugs for patients in the early stages of rheumatoid arthritis.

Single injection CIA models in rats have already been established for pharmacological studies. However, these models have disadvantages in comparison with our model in mice. Firstly, the lower incidence of arthritis $(40 \%-50 \%)^{2}$ makes statistical evaluation of the effects of drugs difficult, and the development and peak (three weeks after the first immunisation) of arthritis are rapid. Recently, the single injection protocol has been developed successfully $(100 \%)$ in DA rats using Freund's incomplete adjuvant. ${ }^{18}$ The same rats, however, developed an acute arthritis after injection with Freund's incomplete adjuvant alone (oil-adjuvant induced arthritis, OAIA). The combination of CIA and OAIA in this model seems to make evaluation of the effects of drugs more complicated.

In conclusion, murine CIA was successfully induced by a single immunisation. Two major considerations regarding the immunisation protocol determine success - namely, the use of Freund's complete adjuvant containing $M$ tuberculosis but not $M$ butyricum as an adjuvant and production using ultrasonication of a high quality antigen emulsion in Freund's complete adjuvant without heating. This CIA model was basically similar to the conventional model in histopathological and pharmacological features. However, the progression of joint inflammation in our model was mild. These findings suggest that this model will be helpful in the discovery and development of novel antirheumatic drugs for patients in an early stage of the disease and also in studying the immune phenomena involved in the development of rheumatoid arthritis. The immunisation conditions used in this CIA model could be widely applicable to development of models of other autoimmune diseases.
1 Sofia R D, Knobloch L C, Vassar H B. Inhibition of the primary lesion of adjuvant induced polyarthritis in rats (18-hour-arthritis test) for specific detection of clinically effective anti-arthritic drugs. F Pharmacol Exp Ther 1975 ; 193: $918-31$.

2 Trentham D E, Townes A S, Kang A H. Autoimmunity to type II collagen: an experimental model of arthritis. $\mathcal{f}$ Exp Med 1977; 146: 857-68.

3 Courtenay J S, Dallman M J, Dayan A D, Marten A Mosedale B. Immunization against heterologous type II collagen induces arthritis in mice. Nature 1980; 283: 666-8.

4 Terato K, Hashida R, Miyamoto K, et al. Histological immunological and biochemical studies on type II collagen-induced arthritis. Biomed Res 1982; 3: 495-505.

5 Wooley P H, Luthra H S, Stuart J M, David C S. T type II collagen-induced arthritis in mice. 1 Majo histocompatibility complex (I region) linkage antibody correlates. $f$ Exp Med 1981 ; 154: 688-700.

6 Phadke K, Fouts R L, Parrish J E, Butler L D. Evaluation of the effects of various anti-arthritic drugs on type II of the effects of various anti-arthritic drugs on type II collagen-induced mouse arthr

7 Kharmacology 1985; 10: 51-60. effects of 3,4-dideoxyglucosone-3-ene, an intermediate in the Maillard reaction. fournal of Agricultural Food Chemistry 1994; 42: 2068-73.

8 Stuart J M, Cremer M A, Townes A S, Kang A H. Type II collagen-induced arthritis in rats: passive transfer with serum and evidence that IgG anticollagen antibodies can cause arthritis. $\mathcal{F} \operatorname{Exp}$ Med 1982; 155: 1-16.

9 Stuart J M, Dixon F J. Serum transfers of collagen-induced arthritis in mice. $\mathcal{F}$ Exp Med 1983; 158: 378-92.

10 Ellis J S, Chain B M, Cooke A, Ibrahim M A, Katz D R. Adjuvant composition determines the induction of type II Adjuvant composition determines the induction of type II collagen-in

11 Seki N, Sudo Y, Yoshioka T, et al. Type II collagen-induced murine arthritis. 1 Induction and perpetuation of arthritis require synergy between humoral and cell-mediated immunity. F Immunol 1988; 140: 1477-84.

12 Germann $\mathrm{T}$, Szeliga J, Hess $\mathrm{H}$, et al. Administration of interleukin 12 in combination with type II collagen induced severe arthritis in DBA/1 mice. Proc Natl Acad Sci USA 1995; 92: 4823-7.

13 Hsieh C S, Macatonia S E, Tripp C S, Wolf S F, O'Garra A, Murphy K M. Development of Th1 CD4+ T cells through IL-12 produced by Listeria-induced macrophages. Science 1993; 260: 547-9.

14 Schmitt E, Hoehn P, Germann T, Rude E. Differential effects of interleukin-12 on the development of naive mouse CD4+T cells. Eur f Immunol 1994; 24: 343-7.

15 Newbould B B. Chemotherapy of arthritis induced in rats by mycobacterial adjuvant. $B r f$ Pharmacol 1963; 21 $127-36$

16 Stuart J M, Townes A S, Kang A H. Nature and specificity of the immune response to collagen in collagen-induced arthritis in mice. $\mathcal{F}$ Clin Invest 1982; 69: 673-83.

17 Wilske K R, Healey L A. Challenging the therapeutic pyramid: a new look at treatment strategies for theumatoid arthritis. F Rheumatol 1991; 17 (suppl 25) 4-7.

18 Holmdahl R, Vingsbo C, Malmstrom V, Jansson L, Holmdahl $M$. Chronicity of arthritis induced with homologous type II collagen (C II) in rats is associated with anti-C II B-cell activation. $\mathcal{f}$ Autoimmun 1994; 7 : 739-52. 